\title{
A general correlation for the stagnation point Nusselt number of an axisymmetric impinging synthetic jet
}

\author{
Tim Persoons ${ }^{a,{ }^{*}}$, Alan McGuinn ${ }^{a}$, Darina B. Murray ${ }^{a}$
}

${ }^{a}$ Department of Mechanical and Manufacturing Engineering, Parsons Building, Trinity College, Dublin 2, Ireland

\begin{abstract}
Whereas the heat transfer mechanisms in steady impinging jets are well understood, the available knowledge of heat transfer to impinging synthetic jets remains inconsistent. This paper provides an objective comparison of the stagnation point heat transfer performance of axisymmetric impinging synthetic jets versus established steady jet correlations. Furthermore, a general correlation for the stagnation point Nusselt number is proposed including the effect of all appropriate scaling parameters: Reynolds number ( $500 \leq R e \leq 1500)$, jet-to-surface spacing $(2 \leq H / D \leq 16)$ and stroke length ( $\left.2 \leq L_{0} / D \leq 40\right)$. Based on the ratio of stroke length to jet-to-surface spacing $L_{0} / H$, four heat transfer regimes are identified.
\end{abstract}

Keywords: impinging jet, forced convection, synthetic jet formation, local heat transfer coefficient, hot-film anemometry

\section{Nomenclature}

\begin{tabular}{|c|c|c|c|}
\hline$D$ & jet orifice diameter or slot width $(\mathrm{m})$ & $T u$ & turbulence intensity $\left(\left(u^{\prime 2}+v^{\prime 2}\right)^{1 / 2} / U_{m}\right)$ \\
\hline$f$ & frequency $(\mathrm{Hz})$ & $U, V$ & axial and radial velocity $(\mathrm{m} / \mathrm{s})$ \\
\hline$H$ & jet-to-surface spacing (m) & $U_{\text {ref }}$ & reference velocity (i.e. mean orifice velocity \\
\hline$h$ & $\begin{array}{l}\text { convective heat transfer coefficient } \\
\left(\mathrm{W} /\left(\mathrm{m}^{2} \mathrm{~K}\right)\right)\end{array}$ & $u^{\prime}, v^{\prime}$ & $\begin{array}{l}\text { for steady jet or } f L_{0} \text { for synthetic jet) }(\mathrm{m} / \mathrm{s}) \\
\text { axial and radial turbulent fluctuation }(\mathrm{m} / \mathrm{s})\end{array}$ \\
\hline$k$ & thermal conductivity of fluid $(\mathrm{W} /(\mathrm{mK}))$ & $x, r$ & axial and radial coordinate $(\mathrm{m})$ \\
\hline$L_{0}$ & synthetic jet stroke length $(\mathrm{m})$ & \multirow{2}{*}{\multicolumn{2}{|c|}{ Greek symbols }} \\
\hline$m, n, p$ & exponents for $\mathrm{Nu}$ dependence on $\mathrm{Re}, \mathrm{Pr}$ & & \\
\hline $\mathrm{Nu}$ & $\begin{array}{l}\text { and } H / D \text { (see Eq. (3)) } \\
\text { Nusselt number }(h D / k)\end{array}$ & $\beta$ & $\begin{array}{l}\text { radial velocity gradient at the stagnation } \\
\text { point }\left((\partial V / \partial r)_{0}\right)\left(s^{-1}\right)\end{array}$ \\
\hline $\operatorname{Pr}$ & Prandtl number & \multicolumn{2}{|c|}{ Subscripts } \\
\hline$q$ & convective heat flux $\left(\mathrm{W} / \mathrm{m}^{2}\right)$ & 0 & properties at stagnation point, or \\
\hline $\operatorname{Re}$ & Reynolds number $\left(U_{\text {ref }} D / v\right)$ & & characteristic scale for synthetic jet \\
\hline Sr & Strouhal number $\left(f D / U_{\text {ref }}\right)$ & $m$ & spatial average in orifice \\
\hline$T$ & temperature $(\mathrm{K})$ & ref & reference temperature \\
\hline$t$ & time (s) & & \\
\hline
\end{tabular}

*Corresponding author.E-mail: tim.persoons@tcd.ie, timpersoons@purdue.edu (T.Persoons).

Present address: School of Mechanical Engineering, Purdue University, 585 Purdue Mall, West Lafayette IN, 47907, USA (Tel.: +1 765494 5638, Fax: +1 765494 0539). 


\section{Introduction}

Impinging jets are a well established technique for achieving high local convective heat transfer rates compared to other single-phase flow configurations. Continuous jets can be used when a pressurized fluid source is readily available e.g. to cool turbine blades or manufacturing processes. As discussed in the following sections, research has shown that beneficial heat transfer regimes are closely linked to strong fluidic perturbations. As such, the effect of flow pulsation has been studied and more recently, zero-net mass flux synthetic jets have been considered for heat transfer applications as well. These jets consist of a propagating train of vortices synthesized from the surrounding fluid by periodic ejection and suction of fluid across an orifice. Synthetic jets are characterized by a Reynolds number, geometric parameters as well as the stroke length $L_{0}=\int_{0}^{1 /(2 f)} U_{m}(t) d t$, where $U_{m}(t)$ is the instantaneous mean orifice velocity.

Some key heat transfer characteristics of impinging jets are reviewed in the following sections, beginning with steady jets, followed by the effect of flow pulsation and finally some established aspects of synthetic jet heat transfer, which is the framework for this paper. The literature review is kept quite broad to allow an objective comparison of the heat transfer performance of impinging steady and synthetic jets (see Sect. 3.1), which is one of the objectives of this work.

\subsection{Heat transfer to a steady jet}

For an incompressible laminar jet with a uniform velocity profile impinging onto a flat surface, the Navier-Stokes equations can be simplified to analytically determine the following expression for the stagnation point heat transfer coefficient [1,2]:

$$
N u_{0}=\frac{h_{0} D}{k}=C\left(\frac{D \beta}{U_{m}}\right)^{1 / 2} \operatorname{Re}^{1 / 2} \operatorname{Pr}^{2 / 5} \text { where } \beta=\partial V /\left.\partial r\right|_{(x, r)=(0,0)}
$$

where $C=0.763$ or 0.570 for an axisymmetric or two-dimensional jet respectively. The radial velocity gradient $\beta$ at the stagnation point can be determined for particular cases. Based on a potential flow analysis, Shadlesky [3] derived $D \beta / U_{m}=3 \pi / 16$ for an axisymmetric jet or $\pi / 4$ for a two-dimensional jet, resulting in

$$
\begin{aligned}
& N u_{0}=0.5856 \operatorname{Re}^{1 / 2} \operatorname{Pr}^{0.4} \text { (for an axisymmetric jet) } \\
& N u_{0}=0.5051 \operatorname{Re}^{1 / 2} \operatorname{Pr}^{0.4} \text { (for a two-dimensional jet) }
\end{aligned}
$$

Throughout this paper, only single jets impinging at 90 degrees onto planar surfaces are considered. Heat transfer to steady jet arrays, oblique jets or jets impinging onto curved surfaces is reviewed elsewhere [4]. Yet even for a simple impinging jet, the heat transfer characteristics depend strongly on the boundary conditions (e.g. the velocity profile in the orifice, turbulence intensity, and geometric confinement) [4]. This explains the wide range of correlations reported in the literature.

Table 1 gives an overview of some stagnation Nusselt number correlations for steady jets. Later in the paper (Table 2), a similar overview is given for synthetic jets. Wherever possible, correlations are recast in a standard formulation 


$$
N u_{0}=c \operatorname{Re}^{m} \operatorname{Pr}^{n}(H / D)^{p}
$$

The correlations in Table 1 are plotted in Fig. 1 as Frössling number $N u_{0} /\left(\operatorname{Re}^{m} \operatorname{Pr}^{n}\right)$ versus $H / D$, with the exponents $m=0.5$ and $n=0.4$ determined from laminar flow theory.

Table 1. Overview of stagnation Nusselt number correlations for a steady jet, impinging perpendicularly onto a flat surface (see Fig. 1)

\begin{tabular}{|c|c|c|c|c|c|}
\hline Source & Jet type & $\begin{array}{l}\text { Instrumentation } \\
\left(D_{s}=\text { spatial }\right. \\
\text { resolution) }\end{array}$ & $H / D$ & $\operatorname{Re}$ & $\begin{array}{l}N u_{0} \text { correlation in } \\
\text { normalized form (Eq. (3)) }\end{array}$ \\
\hline \multirow{2}{*}{$\begin{array}{l}\text { Shadlesky } \\
\text { [3] }\end{array}$} & a) uniform jet ${ }^{(R, U C)}$ & (analytical solution) & & & $0.5856 R e^{1 / 2} \operatorname{Pr}^{0.4}$ \\
\hline & $\begin{array}{l}\text { b) uniform jet } \\
\text { UC) }\end{array}$ & (analytical solution) & & & $0.5051 R e^{1 / 2} \operatorname{Pr}^{0.4}$ \\
\hline $\begin{array}{l}\text { Liu \& } \\
\text { Sullivan [5] }\end{array}$ & $\begin{array}{l}\text { contoured } \\
\text { nozzle }^{(R, U C, Q)}\end{array}$ & $\begin{array}{l}\text { thermo-fluorescent } \\
\text { paint }\left(D_{s} \cong 0.02 D\right)\end{array}$ & $1-2$ & $\begin{array}{l}12270- \\
15100\end{array}$ & $0.585 \operatorname{Re}^{1 / 2} \operatorname{Pr}^{0.4}$ \\
\hline \multirow{2}{*}{$\begin{array}{l}\text { Lytle \& } \\
\text { Webb [6] }\end{array}$} & a) uniform jet ${ }^{(R, U C)}$ & (analytical solution) & $<1$ & & $0.540 \operatorname{Re}^{1 / 2} \operatorname{Pr}^{0.4}(H / D)^{-1 / 2}$ \\
\hline & $\begin{array}{l}\text { b) long pipe } \\
\text { nozzle }^{(R, \cup C, Q)}\end{array}$ & $\begin{array}{l}\text { IR thermography } \\
\left(D_{s}<0.05 D\right)\end{array}$ & $\begin{array}{l}0.25- \\
1\end{array}$ & $\begin{array}{l}3600- \\
27600\end{array}$ & $0.835 \operatorname{Re}^{0.53} \operatorname{Pr}^{0.4}(H / D)^{-0.19}$ \\
\hline $\begin{array}{l}\text { Kataoka } \\
\text { et al. [7] }\end{array}$ & $\begin{array}{l}\text { contoured } \\
\text { nozzle } \\
(R, C, T)\end{array}$ & $\begin{array}{l}\text { thermopile } \\
\left(D_{s} \cong 0.03 D\right)\end{array}$ & $2-10$ & $\begin{array}{l}7000- \\
50000\end{array}$ & $\begin{array}{l}\left(0.82+\mathrm{fn}\left(T u R e^{1 / 2}\right)\right) R e^{1 / 2} \\
\operatorname{Pr}^{1 / 2}\end{array}$ \\
\hline \multirow[t]{2}{*}{$\begin{array}{l}\text { Garimella } \\
\text { \& Rice [9] }\end{array}$} & \multirow[t]{2}{*}{$\begin{array}{l}\text { sharp } \\
\text { orifice }^{(R, C, Q)}\end{array}$} & \multirow[t]{2}{*}{$\begin{array}{l}\text { thermocouples } \\
\left(D_{s}<0.16 D\right)\end{array}$} & $1-5$ & $\begin{array}{l}4000- \\
23000\end{array}$ & $0.462 \operatorname{Re}^{0.585} \operatorname{Pr}^{0.4}(H / D)^{0.024}$ \\
\hline & & & $6-14$ & $\begin{array}{l}4000- \\
23000\end{array}$ & $0.499 \operatorname{Re}^{0.694} \operatorname{Pr}^{0.4}(H / D)^{-0.56}$ \\
\hline $\begin{array}{l}\text { Zumbrunnen } \\
\text { \& Aziz [11] }\end{array}$ & $\begin{array}{l}\text { contoured } \\
\text { nozzle }(2 \mathrm{D}, \mathrm{uC}, \mathrm{Q})\end{array}$ & $\begin{array}{l}\text { thermocouples } \\
\left(D_{s} \cong 0.5 D\right)\end{array}$ & $\cong 7$ & $\begin{array}{l}3100- \\
20750 \\
\end{array}$ & $0.230 R e^{0.589} \operatorname{Pr}^{0.4}$ \\
\hline
\end{tabular}

$R=$ axisymmetric, $2 D=$ two-dimensional, $(U) C=$ (un)confined, $T=$ isothermal heating, $Q=$ constant heat flux

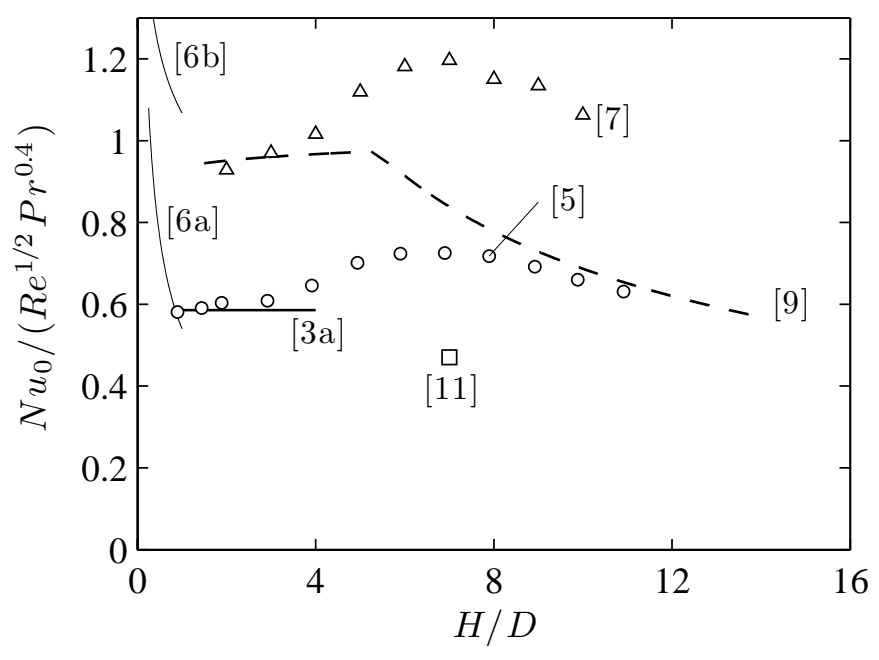

Figure 1. Comparison of stagnation Nusselt number correlations and data reported for steady impinging jets

(see Table 1: - [3a], $\bigcirc[5],-[6 a],[6 b]$ at $R e=3600, \triangle$ [7], $--[9]$ at $R e=4000, \square[11]$ at $R e=3100$ )

Liu and Sullivan [5] have experimentally validated the expressions in Eq. (2), presenting measured values of $N u_{0} /\left(\operatorname{Re}^{1 / 2} \mathrm{Pr}^{0.4}\right)$ as a function of $H / D$ for an axisymmetric low-turbulence jet with a uniform velocity profile. They used a temperature-sensitive fluorescent paint technique on a metal foil heater. The authors note a good agreement with Eq. (2) for $1 D<H<2 D$, a peak heat transfer rate for $6 D<H<8 D$, followed by a decrease in heat transfer for $H>8 D$. 
For an axisymmetric jet at low jet-to-surface spacing $(H / D<1)$, Lytle and Webb [6] estimate the radial velocity gradient $\beta \cong V_{\text {wall }} /(1 / 2 D) \cong U_{m} /(2 H)$, where $V_{\text {wall }}$ is the mean radial velocity at $r=1 / 2 D$. Equation (1) thus yields $N u_{0}=0.5395 \operatorname{Re}^{1 / 2} \operatorname{Pr}^{0.4}(H / D)^{-1 / 2}$, which the authors argue becomes more accurate at lower $H / D$. However, using a long pipe nozzle with fully developed parabolic velocity profile, measured stagnation Nusselt numbers are considerably higher than predicted and exhibit a weaker dependence of $H / D\left(N u_{0}=0.835 \operatorname{Re}^{0.53} \operatorname{Pr}^{0.4}(H / D)^{-0.19}\right)$. In Fig. 1, both the (a) analytical estimate and (b) experimental results are shown.

Viskanta [4] presents a comprehensive literature review for steady jet heat transfer, showing that the values for the Reynolds exponent $m$ in Eq. (3) vary between 0.23 and 0.67 depending on factors such as the nozzle exit velocity profile, where $m=0.5$ is the theoretical value for a uniform laminar flow profile. Non-uniform profiles yield higher heat transfer rates than uniform profiles. This is confirmed in Fig. 1, where sharp orifices and long pipe nozzles typically show higher heat transfer rates than contoured nozzles. For a laminar uniform jet velocity profile, laminar boundary layer theory is expected to hold (Eqs. $(1,2))$ if the stagnation point is in the potential core $(H<4 D)$. Beyond the potential core, free-jet turbulence peaks around $(6 D<H<7 D)$ [7] causing an increase in heat transfer rate in this region. Finally at $H>8 D$, the heat transfer performance deteriorates along with the decaying centreline velocity $[4,5]$.

Kataoka et al. [7] and Hoogendoorn [8] show that turbulence intensity in the jet nozzle can affect $N u_{0}$ by more than $50 \%$, even at low $H / D$. They propose the normalized turbulence intensity $\left(T u R e^{1 / 2}\right.$ ) as an independent scaling quantity in a $N u_{0}$ correlation instead of $H / D$, where $T u=u^{\prime} / U_{m}$. Hoogendoorn [8] measured Tu at $x=0$ in a free jet with the impingement surface removed. Kataoka et al. [7] measured $T u$ at a normal distance of $0.3 D$ from the stagnation point with the impingement surface in place, being a more realistic approach. The relationship between $\left(T u R e^{1 / 2}\right)$ and $H / D$ is discussed by both studies $[7,8]$, yet for comparison some results of $N u_{0}$ as a function of $H / D$ [7] $(10000<R e<50000)$ are included in Fig. 1.

Garimella and Rice [9] studied the geometric effect of a sharp orifice (diameter and length) on the heat transfer performance, resulting in correlations for the stagnation and averaged Nusselt number for a wide range of parameters. As shown in Fig. 1, the non-uniform velocity profiles in a sharp orifice and long pipe nozzle [6] cause a considerably higher heat transfer rate compared to contoured nozzles at the same Reynolds number and jet-to-surface spacing.

In summary, the differences between the steady jet heat transfer correlations shown in Fig. 1 are mainly due to differences in nozzle geometry and flow conditions. These studies for steady jets [2-9] have shown that the heat transfer performance improves for stronger velocity gradients and fluctuations. To achieve higher heat transfer rates, more recent research has focused on exploiting these regimes using pulsating, intermittent and synthetic jets.

\subsection{Heat transfer to a pulsating or intermittent jet}

Liu and Sullivan [5] used low-level fluidic excitation to reinforce the heat transfer enhancement of large scale vortices which form naturally in the jet flow. Using an acoustic speaker mounted upstream inside an axisymmetric contoured nozzle, excitation levels are very small with rms velocity fluctuations $u^{\prime} / U_{m}=\mathrm{O}\left(10^{-4}\right)$. The relative heat transfer enhancement varies between $-10 \%$ and $+10 \%$ 
depending on excitation frequency and $H / D$, however the effect is restricted to the region $1<r / D<$ 2.5 and is not observed in the stagnation region $(0.6<H / D<2, R e=12300)$. This behaviour has been confirmed for a similar geometry $(0.5<H / D<2,10000<R e<30000)$ by O'Donovan and Murray [10].

Zumbrunnen and Aziz [11] studied the heat transfer to an intermittent impinging jet with much higher velocity fluctuation levels using rotating blades $\left(u^{\prime} / U_{m} \cong 1\right)$. Based on laminar flow theory for a two-dimensional impinging jet near the stagnation point, the thermal boundary layer thickness $\delta_{T}(\tau)$ grows to $99 \%$ of the final thickness $\delta_{T}(\infty)$ after a dimensionless time $\tau$ between 4 and $6(0.7<\operatorname{Pr}<$ $15)$, where $\tau=\beta$ t. Based on this theoretical analysis, the authors conclude that heat transfer enhancement due to forced redevelopment of the thermal boundary layer is expected when $\beta / f<3$. This corresponds to a minimum excitation frequency of $S r=f D / U_{m}>1 / 3\left(\beta D / U_{m}\right) \cong 0.262$ for a twodimensional jet or $S r>0.194$ for an axisymmetric jet. This analysis only applies to jets with high excitation levels, not for the small levels used by Liu and Sullivan [5]. Synthetic jets are thought to possess characteristics similar to both low level excited jets and high level pulsed jets, depending on the value of the dimensionless stroke length. This comparison will be further examined in the remainder of this paper.

Zumbrunnen and Aziz [11] present experimental results for the enhancement $N u_{0}{ }^{\text {(puls) }} / N u_{0}{ }^{\text {(steady) }}$ versus dimensionless time $\beta t=\left(\beta D / U_{m}\right) / S r$. An enhancement factor of 2 is noted for $\beta t<2$, while a deterioration in performance is noted for $\beta t>6$ [11]. This minimum Strouhal number criterion seems to be partially confirmed by other sources. Herwig and Middelberg [12] observed increasingly positive enhancement factors from $\mathrm{Sr}>0.029$ for an axisymmetric jet with long pipe nozzle and an upstream pulsator device (up to 1.3 at $S r=0.076$ for square-wave excitation). When extrapolating this analysis to a synthetic jet $\left(L_{0} \propto S r^{-1}\right)$, the above criterion would yield a threshold of $L_{0} / D<5$ to ensure enhancement. While the stroke length $L_{0} / D$ strongly affects the flow field and heat transfer performance of synthetic jets, this particular analogy has not been confirmed.

Although not directly applicable to jet flows, Persoons et al. [13] have demonstrated experimentally that heat transfer enhancement factors up to $N u_{0}^{(\text {puls) }} / N u_{0}{ }^{\text {(steady) }}=1.4$ can be achieved by applying a sinusoidal inline pulsation to a liquid flow microchannel heat sink. Here too, high excitation levels $\left(u^{\prime} / U_{m}>0.2\right)$ are needed to achieve a positive enhancement although the effect of frequency is found to be negligible in the investigated range $(100<R e<650,0.002<S r<0.1,0.002<$ $u^{\prime} / U_{m}<3$ where $U_{m}$ represents the mean channel velocity).

\subsection{Heat transfer to a synthetic jet}

Synthetic jets have been studied extensively for applications in flow control [14]. When impinging onto a heated surface, their highly pulsatile nature promotes entrainment, mixing and break-up of the thermal boundary layers. One of the key advantages over steady and pulsating jets is that synthetic jets require no external supply of pressurized fluid. Several studies of the flow field of free synthetic jets $[14,15]$ have confirmed that $L_{0} / D$ and $R e$ are the appropriate scaling quantities.

For a free axisymmetric synthetic jet, an analytical derivation by Holman et al. [16] predicts a minimum stroke length of $L_{0} / D>0.5$ to generate a vortex which detaches from the orifice and 
propagates far enough to avoid re-entrainment during the suction phase. The threshold is higher $\left(L_{0} / D>\pi\right)$ for a two-dimensional jet [16]. However, there is some variability in the formation thresholds depending on minor geometric details, and experimental studies typically report higher values (e.g. $L_{0} / D>5.5$ for a two-dimensional jet [17] which is confirmed in this study $\left(L_{0} / D>1.5\right.$ for an axisymmetric jet).

For an impinging synthetic jet, the flow field is characterised by the jet-to-surface spacing $H / D$ in addition to $L_{0} / D$ and $R e$. Although some studies (e.g. Valiorgue et al. [18]) have confirmed that $R e$ and the stroke length $L_{0} / D$ are appropriate scaling quantities for an impinging synthetic jet flow, the effect of $L_{0} / D$ is often overlooked in parametric studies. In fact, despite the growing interest in synthetic jet heat transfer, only a few studies report consistent heat transfer characteristics and correlations over a wide range of parameters. This paper will explicitly address this issue.

Initial studies focused only on the effect of certain parameters $\left(L_{0} / D, R e, H / D\right.$, orifice shape) or presented results which are contaminated with mixed parameter effects. For instance, by varying the actuator frequency $f$ at a constant voltage amplitude, both $R e$ and $L_{0} / D$ are affected in different ways. Furthermore, near the Helmholtz resonance frequency appropriate calibration functions should be used to account for fluid compressibility and nonlinear damping in the orifice, especially when studying the behaviour of multiple adjacent jets [19].

Gillespie et al. [20] studied the heat transfer performance of an impinging synthetic jet with a rectangular orifice $(4<H / D<23$ where $D$ represents slot width, $100<R e<400)$. The maximum heat transfer rate occurs for $H$ values of about 14 to 18 slot widths. For larger values, the velocity has decayed too much while for small jet-to-surface spacing, recirculation decreases the performance. Since a fixed frequency was used in this study, the stroke length varies along with the Reynolds number, in an estimated range $6<L_{0} / D<21$.

Pavlova and Amitay [21] present average heat transfer rates, velocity and turbulence intensity distributions for an axisymmetric impinging synthetic jet. The maximum averaged heat transfer rate occurs at $5<H / D<8$ (for $140<R e<740$ ). For the same Reynolds number and $H / D$, two values for the actuation frequency yield about $40 \%$ difference in heat transfer rate, yet this stroke length dependence is not discussed further.

Chaudhari et al. [22,23] present results from a comprehensive study, investigating the effect of a large number of parameters in a wide range $(1000<R e<4000,3<H / D<25$, level of confinement, orifice shape) yet without considering the effect of $L_{0} / D$. All reported Nusselt numbers are averaged over an isothermal heater measuring about $9 D^{2}$ to $64 D^{2}$ in surface area. Both axisymmetric [22] and rectangular [23] orifices are studied, revealing a slightly better performance for rectangular orifices, unlike for steady jets (e.g. Eq. (2)). These studies show that synthetic jets feature a strong Reynolds dependence with exponents in the range $0.8<m<0.9$. In steady jets, similar values of $m \cong 0.8$ are observed in the wall jet $(r / D>1)$ due to stronger turbulence and mixing [24].

Travnicek and Tesar [25] studied the mass transfer characteristics of an annular impinging synthetic jet. By varying the Strouhal number, the authors explicitly investigated the stroke length dependence of the flow field and mass transfer behaviour. Two different flow regimes are identified 
at low and high stroke length, showing a strong influence on the stagnation point mass transfer rate and the overall radial profile of the mass transfer coefficient.

Valiorgue et al. [18] used particle image velocimetry to study the effect of stroke length and Reynolds number for an axisymmetric impinging synthetic jet $\left(H / D=2,500<R e<2150,1<L_{0} / D<\right.$ 22). This study revealed a critical stroke length of $L_{0} / H \cong 2.5\left(L_{0} / D \cong 5\right)$ marking two different flow regimes. At low $L_{0} / H$ (or $H>>L_{0}$ ), the vortices develop yet lose strength before impingement. The stagnation heat transfer rate $N u_{0} /\left(\operatorname{Re}^{m} \operatorname{Pr}^{n}\right)$ increases approximately linearly with $L_{0} / H$. At high $L_{0} / H$ (or $H \ll<L_{0}$ ), the flow tends to an intermittent regime resulting in a time-averaged recirculation vortex. In this regime, the stagnation heat transfer rate becomes independent of $L_{0}$ and only here can $N u_{0}$ be accurately fitted to a correlation of the form of Eq. (3).

The synthetic jet $N u_{0}$ correlations available in the literature $[18,22,23]$ are listed in Table 2 , and are compared to the correlation obtained in the present study in Fig. 5.

\subsection{Objectives}

The aims of this paper are threefold: (i) Firstly, to provide an objective quantitative comparison of the stagnation heat transfer characteristics of synthetic jets versus steady jets. (ii) Secondly, to present a general correlation of stagnation point heat transfer performance for all scaling quantities appropriate for axisymmetric impinging synthetic jets (i.e. Reynolds number, jet-to-surface spacing $H / D$ and stroke length $L_{0} / D$ ), since the effect of $L_{0} / D$ has often been overlooked in heat transfer studies. (iii) Thirdly, to identify heat transfer regimes based on the stroke length dependence. A more detailed analysis of the local heat transfer and fluid dynamic characteristics will be presented in a follow-up paper [26].

\section{Experimental approach}

\subsection{Impinging synthetic jet facility}

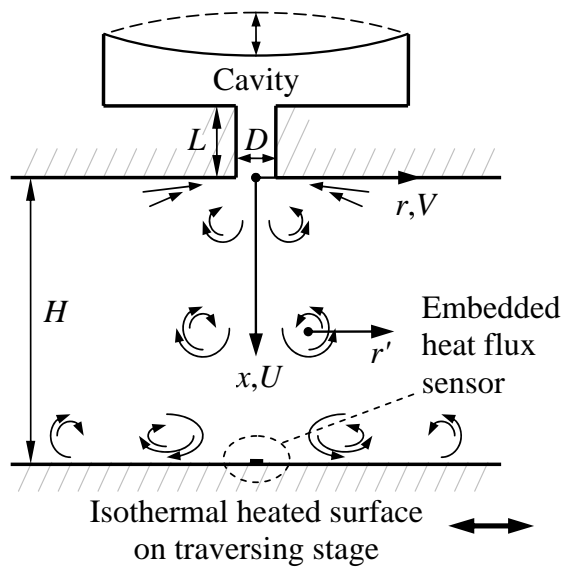

Figure 2. Schematic diagram of the impinging synthetic jet facility

The synthetic jet actuator consists of a polyamide cylindrical cavity $\left(75 \mathrm{~mm}\right.$ diameter, $101.6 \mathrm{~cm}^{3}$ in volume) with a sharp-edged orifice (diameter $D=5 \mathrm{~mm}, 10 \mathrm{~mm}$ long). The bottom of the orifice plate extends outwards over a diameter of $20 D$ to provide a confined outflow condition. A 
loudspeaker (Visaton FR-8, $8 \mathrm{ohm}$ ) is used as a flexible diaphragm actuator. A sinusoidal driving signal is generated by a National Instruments data acquisition system and passed to the loudspeaker via a $40 \mathrm{~W}$ audio amplifier. The cavity is mounted above an instrumented heated surface as shown in Fig.

2. The distance $H$ between orifice exit plane and the heated surface can be adjusted continuously using a vertical traversing stage.

A high-pressure microphone (G.R.A.S. $40 \mathrm{BH}, 0.5 \mathrm{mV} / \mathrm{Pa}$ ) records the pressure in the cavity. This reading is used to adjust the synthetic jet operating point in terms of Reynolds number and stroke length, by applying a pressure-velocity calibration model described by Persoons and O'Donovan [19]:

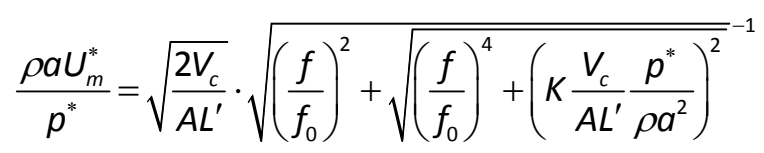

where $\rho$ and $a$ are the density and speed of sound of the fluid, $U_{m}{ }^{*}$ and $p^{*}$ denote the mean orifice velocity and cavity pressure amplitudes, $V_{c}$ is the cavity volume, $A$ and $L^{\prime}$ are the cross-sectional area and effective length of the orifice, and $f_{0}$ is the Helmholtz frequency $\left(f_{0}=a /\left(2 \pi L^{\prime}\right)\left(A L^{\prime} / V_{c}\right)^{1 / 2}\right)$. The empirical model parameter $K$ (representing fluidic damping in the orifice) equals $1.46 \pm 0.13$ for this orifice, as determined in an initial calibration. The model accurately predicts the velocity up to the Helmholtz frequency [19]. It facilitates determination of the Reynolds number $\left(R e=\pi^{-1} U_{m}{ }^{*} D / v\right)$ and

stroke length $\left(L_{0}=\int_{0}^{1 /(2 f)} U_{m}(t) d t=\pi^{-1} U_{m}^{*} / f\right)$ irrespective of changes in actuator performance.

\subsection{Local convective heat transfer measurements}

The synthetic jet impinges onto an isothermal flat copper plate $\left(425 \times 550 \mathrm{~mm}^{2}, 5 \mathrm{~mm}\right.$ thick) [27]. Electrical power is supplied from a DC voltage source to a $1.1 \mathrm{~mm}$ thick silicon rubber mat with embedded wire heaters, glued to the underside of the copper plate. The plate assembly is insulated underneath the heater mat. For typical jet Reynolds numbers, the representative Biot number $h t / k_{c u}$ $\cong 0.0013$ (where $t=5 \mathrm{~mm}$ is the plate thickness). The plate approximates a uniform wall temperature boundary condition, as verified in earlier studies $[10,27]$. The system is typically operated at a fixed surface temperature of $60^{\circ} \mathrm{C}$.

The local convective heat transfer coefficient is defined as $h(=N u k / D)=q /\left(T-T_{\text {ref }}\right)$ where $q$ is the local convective heat flux and $T$ is the local surface temperature. In general, the reference temperature $T_{\text {ref }}$ is the adiabatic surface temperature, i.e. the surface temperature subject to impinging flow without wall heating. Since the flow remains incompressible in these tests $(M a<$ $0.05), T_{\text {ref }}$ equals the jet temperature which is measured using a T-type thermocouple mounted in the jet cavity.

The plate is instrumented with a hot-film sensor (Tao Systems Senflex ${ }^{\mathrm{TM}}, 0.1 \times 1.44 \mathrm{~mm}^{2}$ nickel sensing element on a $51 \mu \mathrm{m}$ thick polyimide substrate). A T-type thermocouple is embedded in the copper plate, directly below the hot-film sensor. The hot-film element is maintained at an overtemperature of $10^{\circ} \mathrm{C}$ with respect to the copper plate using a constant temperature anemometer bridge (Dantec $90 \mathrm{C} 10$ Streamline ${ }^{\mathrm{TM}} \mathrm{CTA}$ ). The local convective heat flux $q$ is correlated to the electrical power dissipation as $q \propto\left(E^{2}-E_{0}^{2}\right) / R_{s}$ where $E$ and $E_{0}$ are the CTA voltage output with and 
without flow respectively, and $R_{s}$ is the sensor resistance in operating conditions. The proportionality factor contains the effective surface area, which accounts for heat conduction loss from the sensor element to its surroundings. An in-situ calibration is performed using a low turbulence steady impinging jet flow (contoured nozzle, $D=13 \mathrm{~mm}, H / D=1$ ) based on the analytical $N u_{0}$ relationship in Eq. (2) derived by Shadlesky [3] for laminar flow theory. The estimated uncertainty on the timeaveraged local Nusselt number depends on the convective heat flux, yet never exceeds $10 \%$ in the investigated parameter range. The hot-film sensor is embedded in a $0.050 \mathrm{~mm}$ deep recess so that it is flush with the surrounding surface. The instrumented plate assembly is mounted on a horizontal traverse system, actuated by a stepper motor to allow automated scanning of a heat transfer coefficient profile. The calibration and measurement methodology is described in detail elsewhere $[10,27,28]$.

For these experiments, the typical Archimedes number $G r / R e^{\prime 2}<0.04$, where the Grashof number $G r$ and $R e^{\prime}$ are defined based on the equivalent width of the heated horizontal surface. The Archimedes number is small enough to neglect buoyancy driven natural convection [29].

\section{Stagnation point heat transfer results}

Using hot-film anemometry as a measurement of the local heat transfer coefficient, this section presents the characteristics of the stagnation point Nusselt number $\mathrm{Nu}$ as a function of Reynolds number $(500 \leq R e \leq 1500)$, jet-to-surface spacing $H(2 \leq H / D \leq 16)$ and stroke length $L_{0}\left(2 \leq L_{0} / D \leq\right.$ 40). Four heat transfer regimes are identified based on the $L_{0} / H$ ratio.

\subsection{Dependence on Reynolds number and jet-to-surface spacing}

Figure 3 shows the Reynolds number dependence of $\mathrm{Nu}_{0}$ for low to moderate jet-to-surface spacing $(2 \leq H / D \leq 10)$ and high stroke length $\left(L_{0} / H>2\right)$. For each Reynolds number, several data points are plotted at different combinations of $H / D$ and $L_{0} / H$. The range excludes the highest jet-tosurface spacings where the jet velocity approaching the impingement region has decayed too much, causing a drop in surface heat transfer rate. A more general correlation as a function of both $R e$ and $H / D$ is presented below. Experiments at low stroke length $\left(L_{0} / H<2\right)$ are excluded for now to ensure a good fit. The dependence on $L_{0}$ is discussed in detail in the following section.

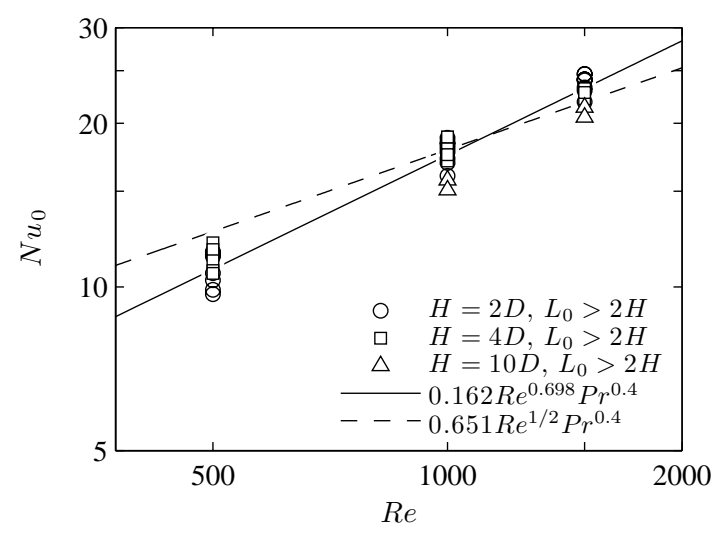

Figure 3. Dependence of stagnation Nusselt number $N u_{0}$ on Reynolds number for moderate jet-to-surface spacing $(2 \leq H / D \leq 10)$ and high stroke length $\left(L_{0} / H>2\right)$ 
The solid line in Fig. 3 represents a least squares power law fit:

$$
N u_{0}=0.1618 \operatorname{Re}^{0.6981} \operatorname{Pr}^{0.4} \quad\left(R^{2}=0.959\right)
$$

where $R^{2}$ represents the coefficient of determination of the least square fit. These experiments were performed only in air ( $P r=0.705)$, yet the Prandtl number dependence is adopted from other sources (Table 1). In spite of the limited range of $R e$ in the present study, the Reynolds exponent $m \cong$ 0.7 agrees well with a steady jet study using a similar geometry [9] (see Table 1). To compare to the behaviour of a laminar steady jet, the dashed line in Fig. 3 represents a fitted power law with the Reynolds exponent forced to $m=1 / 2$.

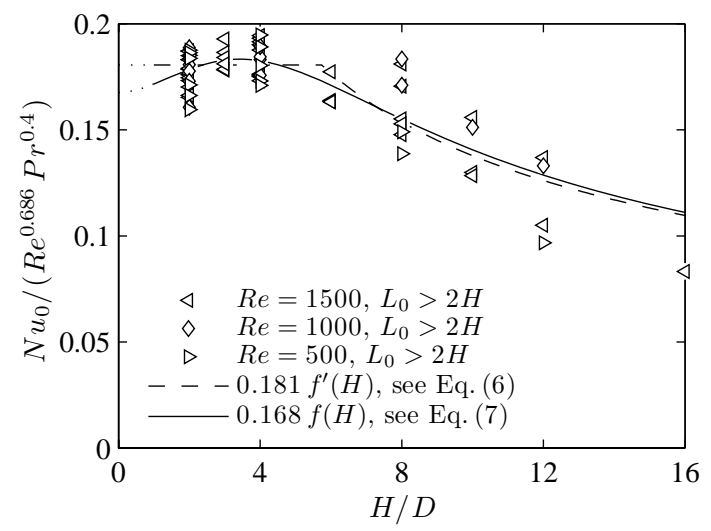

Figure 4. Dependence of stagnation Nusselt number $N u_{0} /\left(\operatorname{Re}^{m} \operatorname{Pr}^{n}\right)$ on jet-to-surface spacing $H / D$ (for $500 \leq R e \leq$ 1500 and $\left.L_{0} / H>2\right)$

Figure 4 shows the dependence of the Frössling number $N u_{0} /\left(\operatorname{Re}^{m} \operatorname{Pr}^{n}\right)$ on the jet-to-surface spacing. As before, experiments at low stroke length are excluded. Two forms of correlation are fitted to the experimental data $\left(2 \leq H / D \leq 16, L_{0} / H>2\right)$. The dashed line in Fig. 4 corresponds to

$$
\begin{aligned}
& N u_{0}=0.1812 \operatorname{Re}^{0.686} \operatorname{Pr}^{0.4} f^{\prime}(H) \quad\left(\mathrm{R}^{2}=0.945\right) \\
& \text { with } f^{\prime}(H)=\max \left(\frac{H / D}{5.74}, 1\right)^{-0.487}
\end{aligned}
$$

and the solid line in Fig. 4 corresponds to

$$
\begin{aligned}
& N u_{0}=0.1676 \operatorname{Re}^{0.686} \operatorname{Pr}^{0.4} f(H) \quad\left(\mathrm{R}^{2}=0.950\right) \\
& \text { with } f(H)=\frac{1+1.108\left(\frac{H / D}{5.21}\right)^{2}}{1+\left(\frac{H / D}{5.21}\right)^{2.487}}
\end{aligned}
$$

For high jet-to-surface spacing, Eq. (7) tends to the same power law relation as Eq. (6), $N u_{0} \propto$ $(H / D)^{-0.487}$. However the formulation of $f(H)$ in Eq. (7) provides a better fit of the peak heat transfer, which occurs at $H / D=3.4$. At this optimal spacing, Eq. (7) reduces to $N u_{0}=0.1833 \operatorname{Re}^{0.686} \mathrm{Pr}^{0.4}$. The Reynolds exponent remains $m \cong 0.7$ similar to Eq. (5). The correction functions $f^{\prime}(H)$ and $f(H)$ tend to unity for small jet-to-surface spacing and tend to a power law $(p=-0.487)$ as $H$ exceeds a threshold value of about $7 D$. 
Table 2 lists Eq. (7) along with other correlations for the stagnation Nusselt number in synthetic impinging jets. Figure 5 shows a general overview of correlations listed in Tables 1-2 for both steady and synthetic impinging jets. The Reynolds exponent for the vertical scale is fixed at $m=1 / 2$ (correcting the Reynolds number dependence where appropriate) to compare against the established correlations for steady jets (see Fig. 1).

Table 2. Overview of stagnation Nusselt number correlations for a synthetic jet, impinging perpendicularly onto a flat surface (see Table 1 and Fig. 5)

\begin{tabular}{|c|c|c|c|c|c|}
\hline Source & Jet type & $\begin{array}{l}\text { Instrumentation } \\
\left(D_{s}=\text { spatial resolution }\right)\end{array}$ & $H / D$ & $R e$ & $\begin{array}{l}N u_{0} \text { correlation in } \\
\text { normalized form (Eq. (3)) }\end{array}$ \\
\hline $\begin{array}{l}\text { Chaudhari } \\
\text { et al. [22] }\end{array}$ & $\begin{array}{l}\text { sharp } \\
\text { orifice }^{(R, C, T)}\end{array}$ & $\begin{array}{l}\text { heater-average } \\
\left(D_{s} \cong 3 . .8 D\right)\end{array}$ & $3-25$ & $\begin{array}{l}1150- \\
4180\end{array}$ & $\underset{(+)}{0.203 \operatorname{Re}^{0.792} \operatorname{Pr}^{0.333}(H / D)^{-0.63}}$ \\
\hline $\begin{array}{l}\text { Chaudhari } \\
\text { et al. [23] }\end{array}$ & $\begin{array}{l}\text { sharp } \\
\text { orifice }^{(2 D, C, T)}\end{array}$ & $\begin{array}{l}\text { heater-average } \\
\left(D_{s} \cong 3 . .8 D\right)\end{array}$ & $3-25$ & $\begin{array}{l}950- \\
4000\end{array}$ & $\underset{(+)}{0.086} \operatorname{Re}^{0.912} \operatorname{Pr}^{0.333}(H / D)^{-0.56}$ \\
\hline $\begin{array}{l}\text { Valiorgue } \\
\text { et al. [18] }\end{array}$ & $\begin{array}{l}\text { sharp } \\
\text { orifice }^{(R, C, Q)}\end{array}$ & $\begin{array}{l}\text { IR thermography } \\
\left(D_{s} \cong 0.03 D\right)\end{array}$ & 2 & $\begin{array}{l}500- \\
2150\end{array}$ & $\begin{array}{l}2.182 \operatorname{Re}^{0.32} \operatorname{Pr}^{0.4} \\
\text { (for } L_{0} / H>2.5 \text { ) }\end{array}$ \\
\hline Present work & $\begin{array}{l}\text { sharp } \\
\text { orifice }^{(R, C, T)}\end{array}$ & $\begin{array}{l}\text { constant temp. hot- } \\
\text { film }\left(D_{s}<0.02 D\right)\end{array}$ & $2-16$ & $\begin{array}{l}500- \\
1500 \\
\end{array}$ & $\begin{array}{l}0.168 \operatorname{Re}^{0.686} \operatorname{Pr}^{0.4} f(H) \\
\text { (for } L_{0} / H>2 \text { ) (Eq. (7)) }\end{array}$ \\
\hline
\end{tabular}

$R=$ axisymmetric, $2 D=$ two-dimensional, $C=$ confined, $T=$ isothermal heating, $Q=$ constant heat flux

+ These correlations [22,23] represent averages over an isothermal heater surface of $40 \times 40 \mathrm{~mm}^{2}$.

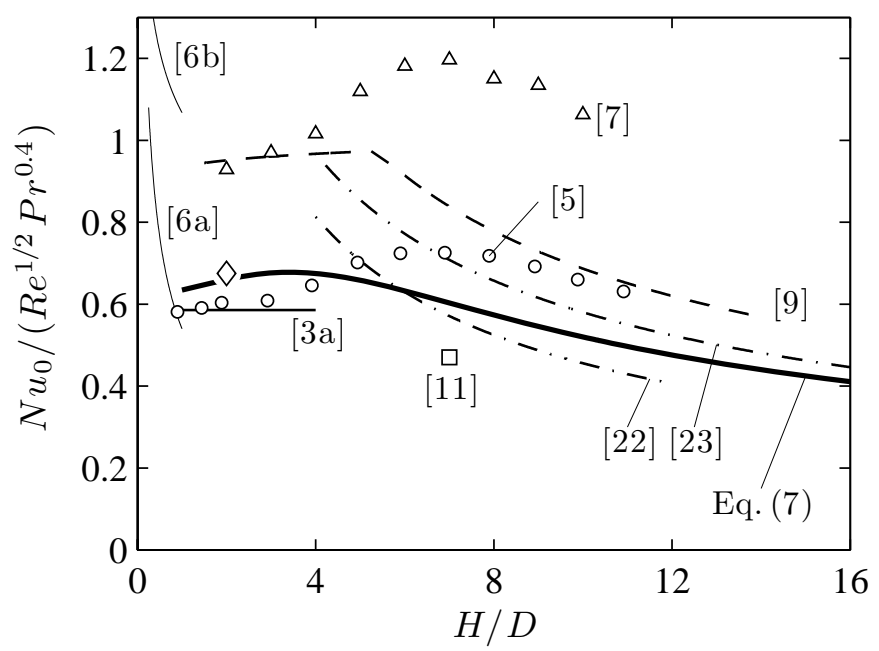

Figure 5. Comparison of stagnation Nusselt number correlations and data reported for steady impinging jets (see Table 1: - [3a], $\bigcirc$ [5], - [6a],[6b] at $R e=3600, \triangle$ [7], - - [9] at $\operatorname{Re}=4000, \square$ [11] at $\operatorname{Re}=3100$ ) and synthetic impinging jets (see Table $2: \diamond[18]$ at $R e=1000,-\cdot-[22,23]$ at $R e=2500$ and Eq. (7) at $R e=1000$ )

At high $H / D$, Eq. (7) agrees reasonably well with other $N u_{0}$ correlations for synthetic jets $[22,23]$ and even steady jets with contoured nozzles $[5,9,11]$. However steady jets with a high turbulence intensity still exhibit a significantly stronger heat transfer [7]. At low $H / D$, Eq. (7) confirms past results by the current authors for $H / D=2$ [18] yet deviates from the results of Chaudhari et al. [22,23]. The reason for this deviation at $H / D<5$ is not clear. For high $H / D$, the correlation by Chaudhari et al. [22] predicts just slightly lower heat transfer values compared to Eq. (7). This might be attributed to the fact that Chaudhari et al. [22,23] present Nusselt values averaged over a $40 \times 40 \mathrm{~mm}^{2}$ isothermal heater surface ( $9 D^{2}$ to $64 D^{2}$ in size) instead of true stagnation point values. The threshold jet-tosurface spacing in Eq. (7) is $H \cong 7 D$, above which the heat transfer decreases as $N u_{0} \propto(H / D)^{p}$. This is 
comparable to steady jets $[5,7,9]$. However the peak heat transfer occurs at a lower jet-to-surface spacing $(H \cong 3.4 D)$ compared to steady jets (typically $6 D<H<8 D[4,5,7,9])$.

\subsection{Dependence on stroke length}

A recent study by the current authors [18] investigated the heat transfer to an impinging synthetic jet for $H / D=2$, revealing two different flow regimes depending on stroke length. A threshold value of $L_{0} / H=2.5$ follows from both flow field and heat transfer results. For large stroke lengths (i.e. small Strouhal number, as $\left.\operatorname{Sr} \propto\left(L_{0} / D\right)^{-1}\right)$, a strong recirculation vortex is observed in the time-averaged flow field at $r / D \cong 2$. Shuster and Smith [15] have shown that the distance travelled by a propagating vortex in a synthetic jet flow scales with stroke length $L_{0}$. As such, it seems like a valid assumption that the ratio $L_{0} / H$ would determine the flow field of an impinging synthetic jet for small values of the stroke length, and thus also affect the heat transfer regime.

The markers in Fig. 6 represent measured values of the Frössling number $N u_{0} /\left(\operatorname{Re}^{m} P r^{n}\right)$ (where $m$ $=0.686$ and $n=0.4$, see Eq. (7)) as a function of $L_{0} / H$ for $H / D=2$. These data agree with the dashed line corresponding to previous results by the authors [18] (see Table 1). However the current instrumentation outperforms the previous approach in terms of spatial resolution, sensitivity and repeatability. As a result of the improved technique, a different trend with a slight peak in heat transfer around $L_{0} / H=5$ is revealed, while still levelling off to a constant at high $L_{0} / H$.

The most significant feature of the data in Fig. 6 is the sudden decrease in heat transfer performance as $L_{0} / H$ falls below 2.5. Valiorgue et al. [18] fitted a linear curve to this region. However, an analytical derivation by Holman et al. [16] predicts that no synthetic jet is formed below a critical value of $L_{0} / D=0.16 \pi \cong 0.5$, since sufficient kinetic energy should be built up during the ejection phase for the vortex to detach from the orifice. Below this formation threshold, the vortex does not propagate away yet is reingested into the orifice in the suction phase. There is some variability on this threshold value e.g. due to geometric details like a minor fillet on the outer orifice edge, and higher values $\left(L_{0} / D\right.$ between 1 and 2$)$ are typically reported in experimental studies $[16,17]$. For a two-dimensional synthetic jet, Fugal et al. [30] even observed a five-fold increase in the critical stroke length (from 2 to 10 slot widths) when using a rounded nozzle exit with a radius of 1 slot width.

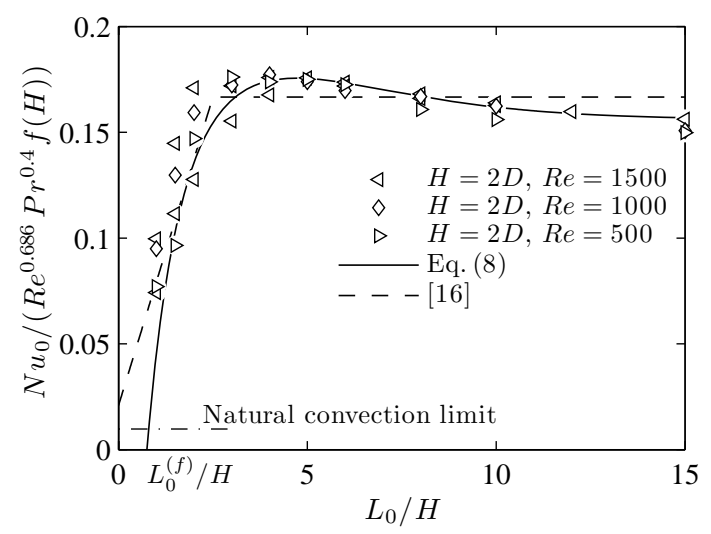

Figure 6. Dependence of stagnation Nusselt number $N u_{0} /\left(\operatorname{Re}^{m} \operatorname{Pr}^{n} f(H)\right)$ on stroke length $L_{0} / H$ for $H=2 D$ (for 500 $\leq R e \leq 1500$ ) compared to correlation by Valiorgue et al. [18] 
In the absence of jet formation the heat transfer performance tends towards the natural convection regime. The typical value of the natural convection Nusselt number for an upward facing horizontal heated surface is represented by the dash-dotted line in Fig. 6 . As such, it seems appropriate to incorporate a formation threshold stroke length $L_{0}^{(f)}$ in the fitted correlation:

$$
\begin{aligned}
& N u_{0}=0.156 \operatorname{Re}^{0.686} \operatorname{Pr}^{0.4} f(H) g\left(L_{0}\right) \quad\left(\mathrm{R}^{2}=0.960\right) \\
& \text { with } g\left(L_{0}\right)=1-e^{-\frac{L_{0}-L_{0}^{(f)}}{0.75 H}}+0.244\left(\frac{L_{0}-L_{0}^{(f)}}{1.80 H}\right)^{2} e^{-\frac{L_{0}-L_{0}^{(f)}}{1.80 H}} \text { and } L_{0}^{(f)}=1.5 D \quad(H / D=2)
\end{aligned}
$$

which corresponds to the solid line in Fig. 6. The formation threshold parameter $L_{0}^{(f)}$ exceeds the theoretical value of $0.5 D[16]$ yet provides the best fit to the experimental data. The function $g\left(L_{0}\right)=$ 0 for $L_{0}=L_{0}^{(f)}$ and tends to unity for $L_{0} \rightarrow \infty$.

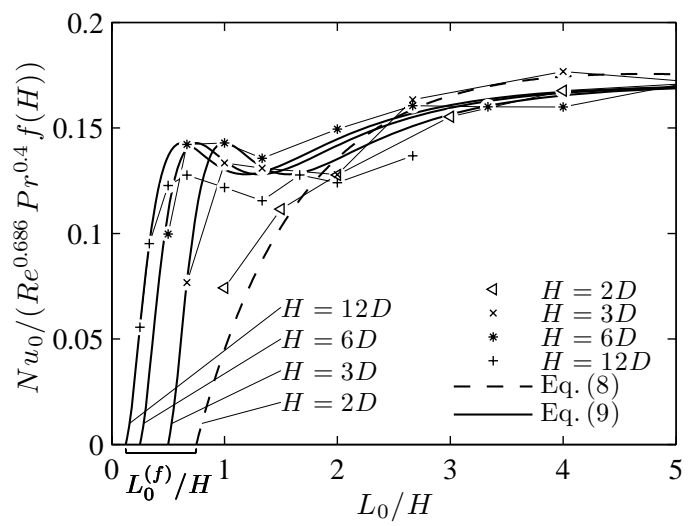

(a)

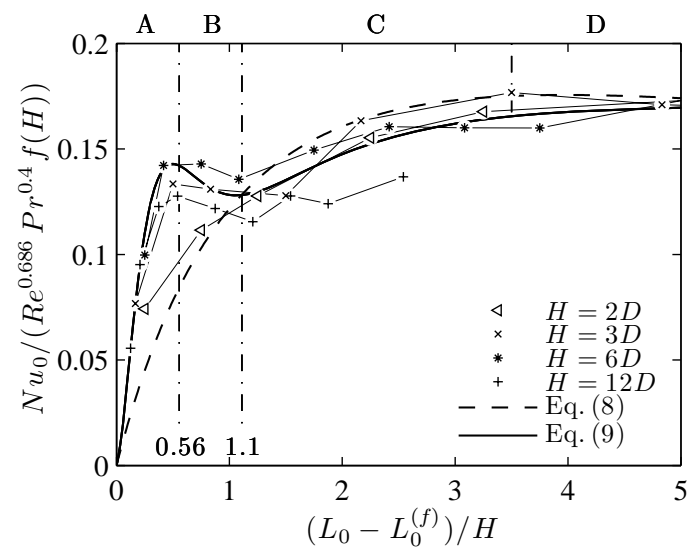

(b)

Figure 7. Dependence of stagnation Nusselt number $N u_{0} /\left(\operatorname{Re}^{m} \operatorname{Pr}^{n} f(H)\right)$ on stroke length (a) $L_{0} / H$ and (b) ( $L_{0}-$ $\left.L_{0}^{(f)}\right) / H$ (for $2 \leq H / D \leq 12, R e=1500$ ) indicating regimes A-D defined in Table 3

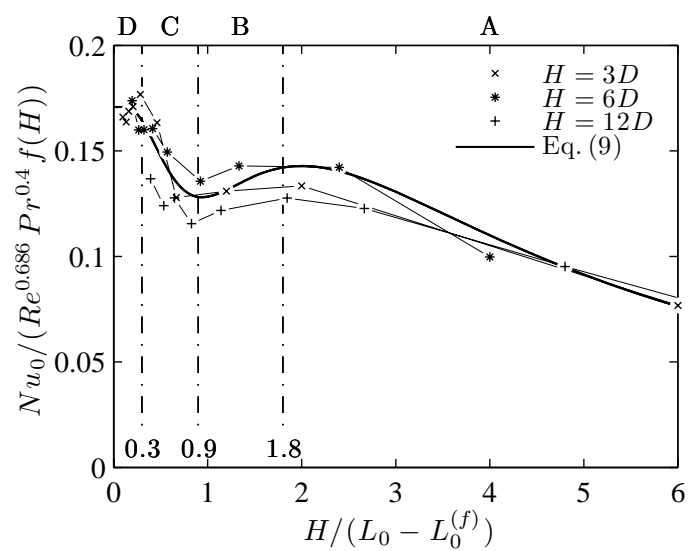

Figure 8. Dependence of stagnation Nusselt number $N u_{0} /\left(\operatorname{Re}^{m} \operatorname{Pr}^{n} f(H)\right)$ on the reciprocal of stroke length $H /\left(L_{0}-\right.$ $\left.L_{0}^{(f)}\right)$ for $3 \leq H / D \leq 12(R e=1500)$ indicating regimes A-D defined in Table 3

Figure 7a presents the results for the entire range of jet-to-surface spacing. Compared to Fig. 6 , the low stroke length behaviour remains present and exhibits the same formation threshold $L_{0}^{(f)}=$ 1.5D. Figure $7 \mathrm{~b}$ presents the same data on a horizontal axis of $\left(L_{0}-L_{0}^{(f)}\right) / H$ instead of $L_{0} / H$, which 
collapses the measurements reasonably well for $3 \leq H / D \leq 12$. There remains a discrepancy with the heat transfer dependence at low jet-to-surface spacing $H / D=2$. The heat transfer peak shifts towards lower $L_{0} / H$ values $\left(\left(L_{0}-L_{0}^{(f)}\right) / H \cong 0.56\right)$ compared to the data for $H / D=2$ in Fig. 6 . After a slight deterioration $\left(0.6<\left(L_{0}-L_{0}^{(f)}\right) / H<1.1\right)$, the heat transfer gradually increases $\left(1.1<\left(L_{0}-L_{0}^{(f)}\right) / H<\right.$ 3.5) and becomes nearly independent of $L_{0}$ for $\left(L_{0}-L_{0}^{(f)}\right) / H>3.5$.

The markers in Fig. 7 represent experiments at $H / D=2,3,6$ and $12(R e=1500)$. The correlation for $H / D=2$ (Eq. (8)) is represented by the dashed line. The solid line corresponds to the following correlation which provides a good fit for $3 \leq H / D \leq 12$ :

$$
\begin{aligned}
& N u_{0}=0.171 \operatorname{Re}^{0.686} \operatorname{Pr}^{0.4} f(H) g\left(L_{0}\right) \quad\left(\mathrm{R}^{2}=0.822\right) \\
& \text { with } g\left(L_{0}\right)=1-e^{-\frac{L_{0}-L_{0}^{(f)}}{1.01 H}}+0.906\left(\frac{L_{0}-L_{0}^{(f)}}{0.187 H}\right)^{2} e^{-\frac{L_{0}-L_{0}^{(f)}}{0.187 H}} \text { and } L_{0}^{(f)}=1.5 D \quad(3 \leq H / D \leq 12)
\end{aligned}
$$

Figure 8 presents the same data as a function of the reciprocal of stroke length, $H /\left(L_{0}-L_{0}^{(f)}\right)$. Since the stroke length is inversely proportional to a Strouhal number $\left(S r^{-1}=L_{0} / D\right)$, this graph shows the frequency dependence of $N u_{0}$. The heat transfer is independent of $L_{0}$ (or $S r$ ) at low frequency ( $L_{0}$ $\gg \mathrm{H})$. As the frequency increases, the heat transfer initially decreases $(0.3<\mathrm{Sr} H / D<0.5)$, followed by a region of increasing heat transfer $\left(0.9<H /\left(L_{0}-L_{0}^{(f)}\right)<1.8\right)$. Finally, heat transfer decreases as the stroke length reduces towards the jet formation threshold.

\subsection{Identified heat transfer regimes}

Similar to Valiorgue et al. [18], heat transfer regimes can be identified based on the value of the stroke length. Figures 6-8 show that at least four heat transfer regimes can be identified for $H / D \geq 3$, however only two are significant for $H / D=2$. Table 3 lists these regimes in order of increasing stroke length, excluding the regime where the stroke length is too small to form a synthetic jet. As shown above, a threshold value of $L_{0}^{(f)}=1.5 D$ is found in this study. No measurements were carried out below this threshold yet the heat transfer is shown to scale directly with $\left(L_{0}-L_{0}^{(f)}\right)$ in the vicinity of the formation threshold. The correlation for the full range of $H / D$ investigated is given by Eq. (10), detailed in section 5 .

For each of the heat transfer regimes identified here, a more detailed investigation of the local heat transfer and fluid dynamic characteristics will be presented in a follow-up paper [26]. The findings are similar to the conclusions drawn by Valiorgue et al. [18] for $H / D=2$. However, a wider parameter range and improvements in the spatial resolution of the measurement technique has enabled a more detailed analysis. Evidence is provided for four distinct regimes (Table 3 ) in terms of heat transfer.

At low stroke length or high jet-to-surface spacing (regime A), the vortices develop yet their strength starts to decay prior to impingement. An overall decrease in heat transfer performance is observed, depending on the level of dissipation of the vortices. By contrast, at high stroke length or low jet-to-surface spacing (regime $\mathrm{D}$ ), the vortices cannot fully develop as they would in a free jet flow, which leads to an intermittent jet regime. In this condition, the heat transfer performance is independent of stroke length, and is only determined by $R e$ and $H / D$. 
Table 3. Heat transfer regimes based on synthetic jet stroke length (see Figs. 6-8)

\begin{tabular}{|c|c|c|c|}
\hline \multirow[t]{3}{*}{ Regime } & \multicolumn{2}{|c|}{ Stroke length thresholds } & \multirow[t]{2}{*}{ Description } \\
\hline & for $H / D=2$ & for $3 \leq H / D \leq 16$ & \\
\hline & $L_{0}<L_{0}^{(f)}=1.5 D$ & $L_{0}<L_{0}^{(f)}=1.5 D$ & $\begin{array}{l}N u_{0} \text { approaches natural convection limit in } \\
\text { absence of synthetic jet formation [16] }\end{array}$ \\
\hline A & $\begin{array}{c}L_{0} / H<2.5 \text { or } \\
\left(L_{0}-L_{0}^{(f)}\right) / H<1.75\end{array}$ & $\left(L_{0}-L_{0}^{(f)}\right) / H<0.56$ & $\begin{array}{l}N u_{0} \text { increases with } L_{0}-L_{0}{ }^{(f)} \text { and reaches a local } \\
\text { maximum }\end{array}$ \\
\hline$B$ & $\begin{array}{c}\text { (not applicable } \\
\text { to } H / D=2 \text { ) }\end{array}$ & $\begin{array}{l}0.6<\left(L_{0}-L_{0}^{(f)}\right) / H<1.1 \\
0.9<H /\left(L_{0}-L_{0}^{(f)}\right)<1.8\end{array}$ & $\begin{array}{l}N u_{0} \text { decreases with } L_{0}-L_{0}{ }^{(f)} \text { (or increases with } \\
\left.H /\left(L_{0}-L_{0}^{(f)}\right) \cong S r H / D\right)\end{array}$ \\
\hline $\mathrm{C}$ & $\begin{array}{c}\text { (not applicable } \\
\text { to } H / D=2 \text { ) }\end{array}$ & $\begin{array}{l}1.1<\left(L_{0}-L_{0}^{(f)}\right) / H<3.5 \\
0.3<H /\left(L_{0}-L_{0}^{(f)}\right)<0.9\end{array}$ & $\begin{array}{l}N u_{0} \text { increases gradually with } L_{0}-L_{0}^{(f)} \text { up to a } \\
\text { constant level }\end{array}$ \\
\hline D & $\begin{array}{c}L_{0} / H>2.5 \text { or } \\
\left(L_{0}-L_{0}^{(f)}\right) / H>1.75\end{array}$ & $\left(L_{0}-L_{0}^{(f)}\right) / H>3.5$ & $N u_{0}$ independent of $L_{0}$ \\
\hline
\end{tabular}

The intermediate range (regimes $\mathrm{B}$ and $\mathrm{C}$, or $0.6<\left(L_{0}-L_{0}^{(f)}\right) / H<3.5$ ) features a local minimum in the heat transfer performance, which is only observed for $H / D \geq 3$. As such, it was not discussed in the previous study for $H / D=2$ [18]. This phenomenon can be attributed to the complex fluid dynamics of impinging synthetic jets. The physics of vortex formation for a synthetic jet are comparable to that of an impulsively started jet. Gharib et al. [31] demonstrate the existence of a universal stroke length in the formation process of a ring vortex. As the stroke length increases, the circulation of the formed vortex increases proportionally. However at a stroke length $L_{0} / D>4$, the vortex circulation levels off to a constant value. As the stroke length increases further, additional fluid ejected from the orifice is not rolled up in the vortex yet starts to form a trailing jet. At high stroke length $\left(8<L_{0} / D<16\right)$, this trailing jet catches up with the vortex and destabilises it, resulting in a decrease in vortex strength. A similar destructive interference has been observed for synthetic jets, which is believed to cause the local decrease in heat transfer performance between regimes $B$ and $C$.

\section{Discussion: Dependence on stroke length or Strouhal number}

Zumbrunnen and Aziz [11] have shown that the heat transfer rate to a pulsating jet increases considerably for sufficiently high Strouhal number $(S r>0.194)$. This behaviour cannot be confirmed for a synthetic jet, and the dependence of $\mathrm{Nu}_{0}$ on Strouhal number appears to be more complex for synthetic jets than for pulsating jets.

For steady impinging jets, Kataoka et al. [7] studied the effect of large-scale coherent structures on the heat transfer in the stagnation region. They noted a dependence of $N u_{0}$ on the flow frequency content, described as $N u_{0} /\left(\operatorname{Re}^{0.5} \mathrm{Pr}^{0.5}\right)=c_{1}+c_{2}(\text { TuRe } \cdot S r)^{0.637}$. The turbulent Reynolds number is defined as TuRe $=u^{\prime} D / v$ (measured at a normal distance of $0.3 D$ from the stagnation point) and the Strouhal number $S r=f D / U_{m}$ corresponds to the frequency of the most energetic eddies in the impingement region. For synthetic jets, these eddies have a well-defined frequency $f$ determined by the jet actuator, and $S r=\left(L_{0} / D\right)^{-1}$. Close to the orifice, synthetic jets have significantly different flow structures than steady jets. However in the far field (from about $x / L_{0}>2$ ) the vortices lose coherence and dissipate into turbulence. In this region, free synthetic jets resemble turbulent steady jets, albeit still with different characteristics [15]. 
For free turbulent steady jets, the turbulence intensity Tu scales approximately linearly with position $x / D$ and levels off beyond a distance of about 6 to 8 diameters from the orifice [7]. Incorporating this assumption in the above expression yields $N u_{0} \propto c_{1}+C_{3}\left(L_{0} / H\right)^{-a}$ for $H / D<6$ and $N u_{0} \propto c_{1}+c_{4}\left(L_{0} / D\right)^{-q}$ for $H / D>8$, with $q=0.637$. The typical order of magnitude of the coefficients $c_{3,4} / c_{1} \cong \mathrm{O}\left(10^{-1}\right)$.

Albeit based on crude approximations, this suggests (i) that the heat transfer performance would improve with decreasing stroke length (or increasing Strouhal number), and (ii) that different scaling quantities are appropriate at low and high $H / D$, respectively $L_{0} / H$ and $L_{0} / D$. The data in Fig. $8 \mathrm{~b}$ does show a region of increasing heat transfer for $0.9<H /\left(L_{0}-L_{0}^{(f)}\right)<1.8$. The subsequent heat transfer deterioration is inherent to synthetic jets as $L_{0}$ approaches $L_{0}^{(\theta)}$.

As shown in Fig. $7 \mathrm{~b}$ and $8 \mathrm{~b}$, the data collapses well when scaled as $L_{0} / H$ in the entire range of $H / D$ investigated, and there is no evidence for $L_{0} / D$ to be the appropriate scaling parameter for high $H / D$. This suggests that the turbulence intensity varies differently with $H / D$ in impinging synthetic jets compared to steady jets, at least for this Reynolds number range. Although information is available for free synthetic jets (e.g. regarding the centreline velocity decay [15]), insufficient knowledge is available for impinging synthetic jets, which could be the focus of future research.

\section{Summary}

A general correlation for the stagnation point heat transfer performance of an impinging synthetic jet is obtained for a wide parameter range, incorporating the effect of Reynolds number (500 $\leq R e \leq 1500)$, jet-to-surface spacing $(2 \leq H / D \leq 16)$ and stroke length $\left(2 \leq L_{0} / D \leq 40\right)$. Combining Eqs. (7-9),

$$
\begin{aligned}
& \text { For } H / D=2: \quad N u_{0}=0.156 \operatorname{Re}^{0.686} \operatorname{Pr}^{0.4} f(H) g\left(L_{0}\right) \quad\left(R^{2}=0.960\right) \\
& \text { and } g\left(L_{0}\right)=1-e^{-\frac{\left.L_{0}-L_{0}^{f}\right)}{0.75 H}}+0.244\left(\frac{L_{0}-L_{0}^{(f)}}{1.80 H}\right)^{2} e^{-\frac{L_{0}-L_{0}^{(f)}}{1.80 H}} \\
& \text { For } 3 \leq H / D \leq 16: N u_{0}=0.171 \operatorname{Re}^{0.686} \operatorname{Pr}^{0.4} f(H) g\left(L_{0}\right) \quad\left(R^{2}=0.822\right) \\
& \text { and } g\left(L_{0}\right)=1-e^{-\frac{\left.L_{0}-L_{0}^{f f}\right)}{1.01 H}}+0.906\left(\frac{L_{0}-L_{0}^{(f)}}{0.187 H}\right)^{2} e^{-\frac{L_{0}-L_{0}^{(f)}}{0.187 H}} \\
& \text { with } L_{0}^{(f)}=1.5 D \text { and } f(H)=\left(1+1.108\left(\frac{H / D}{5.21}\right)^{2}\right) /\left(1+\left(\frac{H / D}{5.21}\right)^{2.487}\right)
\end{aligned}
$$

The above correlation matches more than $85 \%$ of the experimental data in the entire parameter range to within $\pm 15 \%$, as shown in Fig. 9. Most of the outliers are for the highest jet-to-surface spacing $(H / D=16)$. The overall rms normalized deviation $\delta N u_{0}=11.2 \%$, defined as $\delta N u_{0}=\left[n^{-1}\right.$ $\left.\sum\left(\left(N u_{0, \text { cor }}-N u_{0, \text { exp }}\right) / N u_{0, \text { exp }}\right)^{2}\right]^{1 / 2}$.

For high stroke length (regimes $C$ and $D$ in Table 3: $L_{0} / H>2.5$ for $H / D=2 ;\left(L_{0}-L_{0}^{(f)}\right) / H>1.1$ for 3 $\leq H / D \leq 16)$, Eq. (10) becomes reasonably independent of $L_{0}$. In this case the general $N u_{0}$ correlation in Eq. (10) collapses well to Eq. (7) shown in Fig. 5 . Figure 5 provides an objective comparison of the 
stagnation point heat transfer performance of synthetic jets versus steady jets. Synthetic jets achieve a comparable performance to steady impinging jets with a low turbulence level (e.g. low Reynolds number jets using a contoured nozzle) $[5,9,11]$. However, steady jets with a high turbulence intensity (e.g. using a sharp orifice) still exhibit significantly higher heat transfer rates [7].

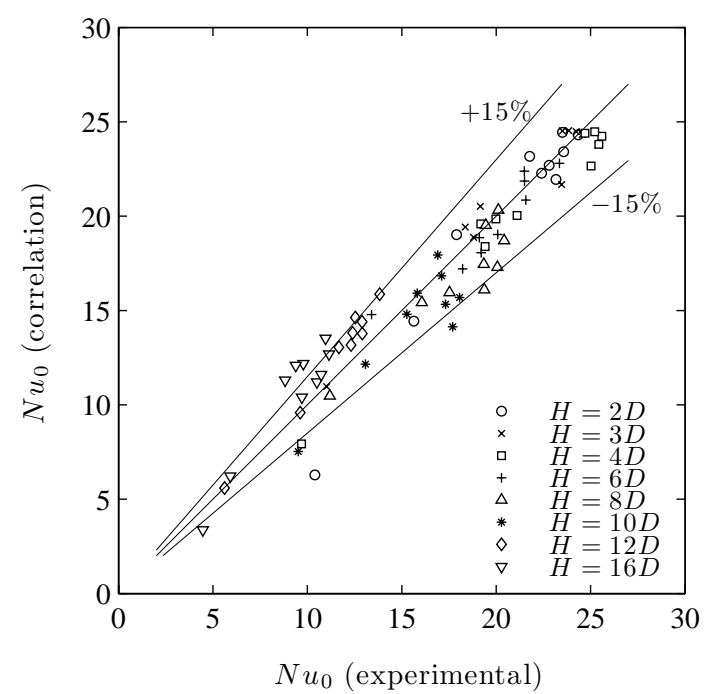

Figure 9. Agreement of stagnation Nusselt number $N u_{0}$ between experimental data and correlation in Eq. (10) for the entire investigated parameter range $\left(500 \leq R e \leq 1500,2 \leq H / D \leq 16,2 \leq L_{0} / D \leq 40\right)$

The high Reynolds exponent ( $m \cong 0.686)$ and the behaviour at high jet-to-surface spacing $\left(N u_{0} \propto\right.$ $\left.(H / D)^{-0.487}\right)$ compares well with other synthetic jet studies [22,23], and even compares well to the behaviour in the turbulent wall jet region of steady jets [24]. The jet-to-surface spacing for maximum heat transfer $(H / D=3.4)$ is smaller than the value observed for steady jets $(6<H / D<8)[4,5,7,9]$.

However, Fig. 5 does not account for the stroke length dependence, which is inherent to the heat transfer and fluid dynamics of impinging synthetic jets. This work has identified four heat transfer regimes (Table 3 ) based on the ratio of stroke length to jet-to-surface spacing $L_{0} / H$. This is considered to be an appropriate dimensionless group to identify flow and heat transfer regimes in impinging synthetic jets, since the stroke length (along with the Reynolds number) is an appropriate scaling quantity for the fluid dynamics of synthetic jets [15].

For flow control applications, Shuster and Smith (2007) concluded that low stroke length regimes should be avoided to maximize the far field momentum flux of a synthetic jet. Similarly for heat transfer applications, the low stroke length regime (A) should be avoided (see Table 3 ) to ensure good heat transfer performance. For the same Reynolds number, the relative difference in terms of stagnation Nusselt number between the other three regimes (B-D) is about $30-40 \%$. Peak heat transfer performance at a given Reynolds number is obtained in regime $D$, for a high ratio of stroke length to jet-to-surface (i.e. low frequency and low $H / D$ ).

Although not discussed in this paper, the energy efficiency is maximized by operating the jet at the resonance frequency of the actuator and jet cavity. This becomes an important consideration when designing small-scale synthetic jet actuators e.g. for cooling portable electronics. Downsizing the actuator leads to an increase in resonance frequency, which means that high stroke length (i.e. 
low frequency) operation becomes inefficient. In this case, reaching optimal heat transfer performance could be achieved by taking advantage of the local $N u_{0}$ maximum between regimes $\mathrm{A}$ and $B$ at $\left(L_{0}-L_{0}^{(f)}\right) / H \cong 0.6$ (for $H / D \geq 3$ ).

Based on the heat transfer regimes identified in this work, a more detailed analysis of the local heat transfer and fluid dynamic characteristics will be presented in a follow-up paper [26].

\section{Acknowledgements}

Dr. Tim Persoons is a Marie Curie research fellow of the Irish Research Council for Science, Engineering and Technology (IRCSET). The authors acknowledge the financial support of Science Foundation Ireland (Grant no. 07/RFP/ENM123).

\section{References}

1 H. Schlichting, Boundary Layer Theory, fourth ed., McGraw-Hill, New York, 1960, pp. 78-83.

2 M. Sibulkin, Heat transfer near the forward stagnation point of a body of revolution, Journal of the Aeronautical Sciences (1952) 570-571.

3 P.S. Shadlesky, Stagnation point heat transfer for jet impingement to a plane surface, AIAA Journal 21 (1983) 1214-1215.

4 R. Viskanta, Heat-transfer to impinging isothermal gas and flame jets, Experimental Thermal and Fluid Science 6 (2) (1993) 111-134.

5 T. Liu, J.P. Sullivan, Heat transfer and flow structures in an excited circular impinging jet, International Journal of Heat and Mass Transfer 39 (17) (1996) 3695-3706.

6 D. Lytle, B.W. Webb, Air jet impingement heat transfer at low nozzle-plate spacings, International Journal of Heat and Mass Transfer 37 (12) (1994) 1687-1697.

7 K. Kataoka, R. Sahara, H. Ase, T. Harada, Role of large-scale coherent structures in impinging jet heat transfer, Journal of Chemical Engineering of Japan 20 (1) (1987) 71-76.

8 C.J. Hoogendoorn, Effect of turbulence on heat-transfer at a stagnation point, International Journal of Heat and Mass Transfer 20 (12) (1977) 1333-1338.

9 S.V. Garimella, R.A. Rice, Confined and submerged liquid jet impingement heat transfer, Journal of Heat Transfer-Transactions of the ASME 117 (4) (1995) 871-877.

10 T.S. O'Donovan, D.B. Murray, Effect of acoustic excitation on the heat transfer to an impinging air jet, in: Proceedings of the ASME-JSME Thermal Engineering Summer Heat Transfer Conference, Vancouver, BC, Canada, 2007, HT2007-32800.

11 D.A. Zumbrunnen, M. Aziz, Convective heat-transfer enhancement due to intermittency in an impinging jet, Journal of Heat Transfer-Transactions of the ASME 115 (1) (1993) 91-98.

12 H. Herwig, G. Middelberg, The physics of unsteady jet impingement and its heat transfer performance, Acta Mechanica 201 (1-4) (2008) 171-184.

13 T. Persoons, T. Saenen, T. Van Oevelen, M. Baelmans, Effect of flow pulsation on the heat transfer performance of a microchannel heat sink, Journal of Heat Transfer-Transactions of the ASME (in review)

14 A. Glezer, M. Amitay, Synthetic jets, Annual Review of Fluid Mechanics 34 (2002) 503-529.

15 J.M. Shuster, D.R. Smith, Experimental study of the formation and scaling of a round synthetic jet, Physics of Fluids 19 (2007) 045109. 
16 R. Holman, Y. Utturkar, R. Mittal, B.L. Smith, L. Cattafesta, Formation criterion for synthetic jets, AIAA Journal 43 (2005) 2110-2116.

17 B.L. Smith, G.W. Swift, Synthetic jets at large Reynolds number and comparison to continuous jets, in: Proceedings of the 31st AIAA Fluid Dynamics Conference, Anaheim, CA, USA, 2001, paper 2001-3030.

18 P. Valiorgue, T. Persoons, A. McGuinn, D.B. Murray, Heat transfer mechanisms in an impinging synthetic jet for a small jet-to-surface spacing, Experimental Thermal and Fluid Science 33 (4) (2009) 597-603.

19 T. Persoons, T.S. O’Donovan, A pressure-based estimate of synthetic jet velocity, Physics of Fluids 19 (12) (2007) 128104.

20 M.B. Gillespie, W.Z. Black, C. Rinehart, A. Glezer, Local convective heat transfer from a constant heat flux flat plate cooled by synthetic air jets, Journal of Heat Transfer-Transactions of the ASME 128 (10) (2006) 990-1000.

21 A. Pavlova, M. Amitay, Electronic cooling using synthetic jet impingement, Journal of Heat TransferTransactions of the ASME 128 (9) (2006) 897-907.

22 M. Chaudhari, B. Puranik, A. Agrawal, Heat transfer characteristics of synthetic jet impingement cooling, International Journal of Heat and Mass Transfer 53 (2010) 1057-1069.

23 M. Chaudhari, B. Puranik, A. Agrawal, Effect of orifice shape in synthetic jet based impingement cooling, Experimental Thermal and Fluid Science 34 (2010) 246-256.

24 K. Jambunathan, E. Lai, M.A. Moss, B.L. Button, A review of heat-transfer data for single circular jet impingement, International Journal of Heat and Fluid Flow 13 (2) (1992) 106-115.

25 Z. Travnicek, V. Tesar, Annular synthetic jet used for impinging flow mass-transfer, International Journal of Heat and Mass Transfer 46 (17) (2003) 3291-3297.

26 T. Persoons, A. McGuinn, R. Farrelly, D.B. Murray, Convective heat transfer mechanisms for an axisymmetric impinging synthetic jet (in preparation for submission to Int J Heat Mass Transfer).

27 T.S. O'Donovan, D.B. Murray, Jet impingement heat transfer - Part I: Mean and root-mean-square heat transfer and velocity distributions, International Journal of Heat and Mass Transfer 50 (2007) 3291-3301.

28 T.S. O’Donovan, T. Persoons, D.B. Murray, High resolution hot film measurement of surface heat flux to an impinging jet, Meas Sci Technol (in review).

29 F.P. Incropera, D.P. DeWitt, T.L. Bergman, A.S. Lavine, Fundamentals of Heat and Mass Transfer, sixth ed., John Wiley \& Sons, 2007, pp. 593-594.

30 S.R. Fugal, B.L. Smith, R.E. Spall, Displacement amplitude scaling of a two-dimensional synthetic jet, Physics of Fluids 17 (2005) 045103.

31 M. Gharib, E. Rambod, K. Shariff, A universal time scale for vortex ring formation, Journal of Fluid Mechanics 360 (1998) 121-140. 